\title{
Live Role-Playing Games: Implications for Pervasive Gaming
}

\author{
Jennica Falk ${ }^{1}$ and Glorianna Davenport ${ }^{2}$ \\ ${ }^{1}$ PLAY - Interactive Institute, Göteborg, Sweden \& Media Lab Europe, Dublin, Ireland \\ jennica.falk@tii.se \\ ${ }^{2}$ Interactive Cinema - MIT Media Laboratory, \\ Massachusetts Institute of Technology, Cambridge, Massachusetts \\ gid@media.mit.edu
}

\begin{abstract}
Live role-playing (LRP) games stand as powerful metaphorical models for the various digital and ubiquitous forms of entertainment that gather under the term pervasive games. Offering what can be regarded as the holy grail of interactive entertainment - the fully immersive experience - LRP games provide a tangible and distributed interface to a gaming activity that is emergent, improvised, collaboratively and socially created, and have the immediacy of personal experience. Supported by studies of LRP games, specifically aspects of costume, set design and props, we outline the interface culture specific to LRP and in which ways this culture may inform the design of pervasive games.
\end{abstract}

\section{Introduction: Pervasive Games}

Pervasive games make up a relatively contemporary area for academic inquiry. These are digital games that move beyond the traditional computer interfaces and into the physical world to occupy time and place on a human scale. These post-desktop games inhabit our physical surrounding and objects within it, employing human senses in ways that differ greatly from that of other electronic games. They take on ubiquitous and tangible forms - properties that contribute to the blurring of the lines between player and game character, game world and real world, and game artifacts and real world objects. These games have matured into experiences of personal immediacy, granting privilege to the skills with which we act and interact in our physical environment. 'Pirates!' is an early example of such a game. It is a multi-player game played on networked handheld computers equipped with radio-frequency proximity sensors. The sensors make it possible to detect the players' relative position within a physical space, which they must navigate in order to explore the game world that unfolds on the computer screen [1]. In the M-Views project at MIT Media Laboratory's Interactive Cinema group, researchers are creating content and technology for what can be described as narrative treasure hunts. Equipped with handheld media machines, users navigate triangulated WLAN areas to follow movie characters around in a narrative taking place in the physical world [11]. This mobile cinema delivers motion picture stories based on the users' absolute location in time and space, catering for the participation of an audience immersed in context-aware multimedia. Blast 
Theory's gaming events Can You See Me Now? and I Like Frank explore the hybrid space between virtual and physical worlds through a mixed reality approach [2], employing human engagement in the physical world as an interaction mode for pervasive game play.

Designers of pervasive games face a number of challenges, most of which they share with designers of other ubiquitous computing applications. Notable are issues of technological nature, such as how to enable context-awareness and content delivery and issues of social nature; the impact of the application on society, privacy, and its integration with public spaces; issues of designing unambiguous functionality and how to interface content, and so forth. It is the subject matter of interfaces that we are currently focusing on; studying the interface model of live role-playing ( $L R P)$ games. This paper describes the nature of LRP, reports on observations from a set of games, and suggests implications for the design of ubiquitous game interfaces.

\section{Live Role-Playing Games: Definitions}

It is not straightforward to define what live role-playing games are - there are likely as many definitions as there are games. As Daniel Mackay observes about roleplaying games, "each [game] is performed in a different way, and the performances that result are circumscribed by different boundaries." [8]. This is not necessarily an unfortunate thing but rather a good indication that the game genre offers great depth and variety in style to players. However, the situation calls for our own definition to be articulated. The following definition is applicable to the games we have studied in the past few years:

A live role-playing game is a dramatic and narrative game form that takes place in a physical environment. It is a story-telling system in which players assume character roles that they portray in person, through action and interaction. The game world is an agreed upon environment located in both space and time, and governed by a set of rules - some of which must be formal and quantifiable.

Purposefully uncomplicated, our definition highlights a number of features of special importance, which require some extra attention. Firstly, the definition indicates that LRP is about gaming. Like other games, they have a system of rules, context for advancement and goals, as well as obstacles and threats to those goals. This makes LRP different from some deceivingly similar activities, specifically living history and re-enactment events that may provide the same sort of spectacle but not the formal rules system. Secondly, they are also story-telling systems. The collective and individual game activities both have narrative qualities with emergent, collaborative, and mostly unpredictable outcomes. In this aspect LRP displays similarities with improvisational theatre, with the important difference that live role-playing games are devoid of the audience concept. Thirdly, LRP games take place in physical environments, bound by specific locations and time-frames. This is of course directly relevant to a discussion on how the physical world can be said act as an interface to the game, but more specifically it offers an appropriate metaphor for researching design implications for pervasive games.

Janet Murray describes live role-playing games as "games in which [fans of fantasy literature] assume the roles of characters within the same fictional universe."[10]. Further she elaborates, "players share a sense of exploring a common fictional land- 
scape and inventing their stories as they go along." Her definition draws attention to an active audience literary genre and the dramatic exploration of a narrative reality.

It should be said that even if some definitions of LRP games (including our own) emphasize their game nature, this is not a widely accepted categorization. In the Dogma 99 [15] manifesto, Lars Wingård and Eirik Fatland argue that it is a "form and a method of individual and collective expression", that it is a medium rather than a genre and a "meeting between people, who through their roles, relate to each other in a fictional world." Their definition calls attention to LRP as performing arts, centering on the characters and what happens to them.

Mike Pohjola further supports the notion of active participation with his statement, "the creative side and the receptive side are no longer separate. The experience of role-playing is born through contributing. No-one can predict the events of a session beforehand, or recreate them afterwards." [12]. His statement reflects the improvisational nature of LRP games, while also emphasizing that it is very difficult (if not impossible) to tell pre-determined stories through LRP.

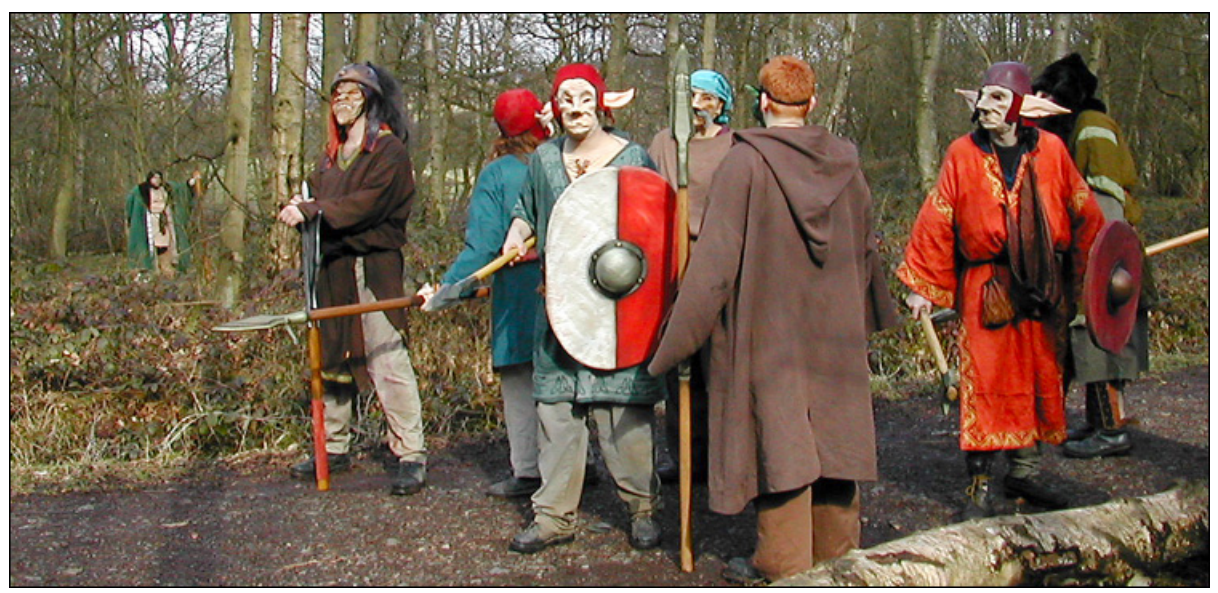

Fig. 1. Live role-playing games take place in the physical world

One principal objective of LRP games is the dramatization of a make-believe world. Offering social and emergent narrative co-creation, they encourage and depend on the players' active commitment and inter-personal participation. Daniel Mackay captures some of its essence, pointing to its peripatetic style: "The live action roleplaying game is distinguished by the players' bodily movement through space and their assumption of costumes and other tools or techniques of naturalistic theatre. The live-action role-playing is both an environmental and improvisational performance." [8, p. 182].

All LRP games of our definition take place within an agreed upon theme or narrative setting that provides the context for players' actions and character roles. This theme can be pretty much anything you can imagine; the possibilities are inexhaustible. To give some obvious and common examples, it may be fantasy and involve magic, heroes, and fantastic creatures, much like the game world settings of the classic tabletop role-playing games. Or, it may be realistic, based on historic events or contemporary life. Or it can be futuristic post apocalyptic environments, involving 
mutants and cyborgian characters dwelling in deserted cities. Or, it can be any combination of these! Whatever the theme, the players will act on and within that context, creating their characters' costume, background stories, personalities and ambitions that fit into and contribute to it.

\subsection{Live Role-Play and Theatre}

It is possible to draw parallels between LRP and theatre, at least metaphorically. One can liken the physical environment in which the role-play takes place to the theatre's stage, upon which the players are actors and where the use of props and set-design enhances and supports the interplay between them. However, a critical difference between LRP and theatre fails the comparison. LRP games are devoid of the audience concept. It may seem an unlikely detail, especially since it is a spectacle coming across hundreds of people in costume and fake weapons behaving in mysterious ways. It is probably true that LRP is quite a show for some part of the display, but it is likely to end there. It is immensely difficult to get anything more than a flavor of what is actually going on during a LRP event, and trying to grasp or capture the story as an outsider would be an impressive feat. The most important reason for this is the participatory nature of the event. The story emerges out of the active participation of and interaction between characters. The only way to experience it with some level of coherency is really to be personally involved. From a narrative perspective, this is why LRP is different from for historical re-enactment events.

\subsection{Why Do People Play LRP Games?}

It is beyond the scope of this paper to analyze, or even speculate, why people play LRP games. There are many sociological studies that are devoted to this subject primarily in the case of table-top role-playing games [e.g. 4, 8] - that suggest reasons ranging from escapism and the safe exploration of anti-social behavior (such as violence) to the need for pure and simple fun. This is certainly true also in live roleplaying games. For the purposes of our work however, we direct our attention to the game mechanisms - that is, mechanisms that have little to do with the personality of the individual player - that enable immersion and engrossment within the game and its narrative theme. In other words, we are interested in how the very design and setup of the game play affects players' commitment and engagement.

\section{The 'Lorien Trust' Game World}

We have followed live role-players in the United Kingdom for two years, primarily in the Lorien Trust (LT) LRP system [7]. During the summer months each year, LT organizes four main events at Locko Park in Derbyshire, each of which attracts thousands of players. We participated in a total of four of the main LT events during 2002 and 2003, each of which engaged between approximately 3500 and 4500 people. We also participated in a number of smaller events (25-100 participants) sanctioned by the LT. Each of these events typically takes place over the course of four days of 
continuous game play. Each event is part of a longer campaign that has been running for over ten years. In this study we are looking specifically at aspects of costume, physical props and set design with the aim to examine how they support or interface with the game play. The following analysis, although based on observations in the LT system, is not intended to be specific to this system; however, it should be noted that aspects of our analysis may not always be applicable to LRP games in general.

\subsection{Costume as Interface}

In mask theatre, actors act with masks covering their faces. To the actors, the masks work as tools of transformation into an often trance-like state of mind where they are not simply acting with a mask on, but are "possessed by the mask" [6, pp. 143-146]. In many ways, costume has the same effect on a live role-player, allowing them to enter into a persona separate and often different from themselves but yet one that is an extension of their selves. The LRP player, like a stage actor, is a person who undergoes a transformation into a character. The character's costume and accessories, or kit, aids this transformation, functioning as an interface on a number of different levels. First, it is an interface between the player and the character, i.e. it is something to 'get into character' with. The following anecdote illustrates this interface function of costume and kit. Player A is asking player B to change character from the one he is currently playing to make a brief appearance as another character:

A: "Can you bring him into play?"

B: "Nah, I didn't bring any of his kit."

A: "Don't worry about it. There should be lots of stuff you can borrow."

B: "You don't understand, I can't be, and don't want to be him without his stuff."

What A and B disagree on is the importance of costume as a tool for transformation. On the one hand, B feels that it would be a disservice to his character to portray it without the signature apparel, and on the other hand he suggests that it would be difficult to do even if he decided to try. It would be like expecting Charlie Chaplin to be The Tramp without the baggy pants, the too tiny jacket, the too big shoes, the hat and the stick. It would not be The Tramp and Chaplin would not be able to - nor would he likely want to - portray him!

Costume is also an interface between players. Consider the following dialogue:

A: "Where is Bray?" (Bray is the name of player B's character)

B: "She is in my bag still. I haven't had a chance to get into kit..."

A: "Well, go get her! I need to talk to her!!"

Here, A has already transformed into his character, while B has not. A feels that B needs to get into her character so that he can interact with it. It is not sufficient that B is there in the capacity of a playing person - she must become the character. A as a character is somehow unwilling to relate to the B unless she is Bray. This is likely directly linked to the fact that as long as you are 'out of character', i.e. if you are the person and not the character, the things you say, hear, see or do has no meaning within the game context. Only the character's actions and experiences are sanctioned and considered to be true. Costume signals when a person has entered into the mode of playing the game and therefore is eligible "prey" so to speak. In this capacity costume both contextualizes and endorses players' actions and behavior. 


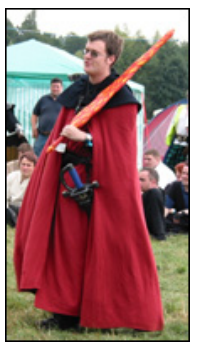

(2)

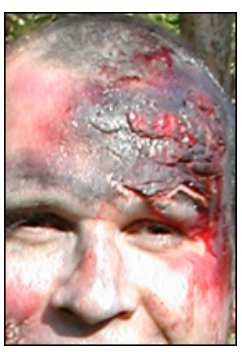

(3)

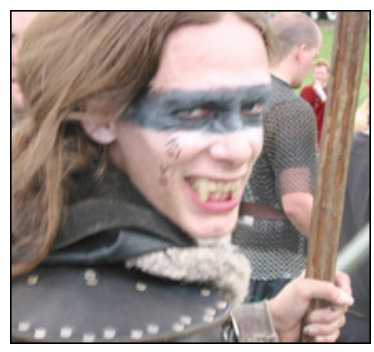

(4)

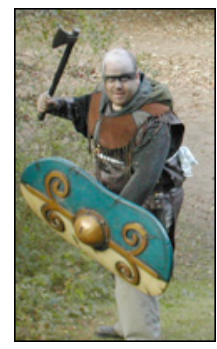

(5)

Fig. 2.-5. Samples of costume and personal props such as weapons and make-up

\subsection{Set-Design and Props as Interface}

The integration of game space and physical space creates a graspable game environment, the stage on which the game takes place. Physical structures may be used as game locations, and sometimes even purposely constructed to enhance the game world. This physicality contributes to creating a highly immersive, tangible and location-specific interface to the game. Allowing the game to extend in this way into the real world fosters coherent and meaningful role-playing relationships between characters and the game world. Figure 7 shows a library setting, which players would have to visit to gain some specific knowledge. In figure 8 is a magic mushroom that is one out of seven or eight like it, positioned in a circle on a field. The mushrooms form a transportation circle - an LT specific apparatus used to move characters great distances, and from which monsters are cloned and poured into the game world ${ }^{1}$.

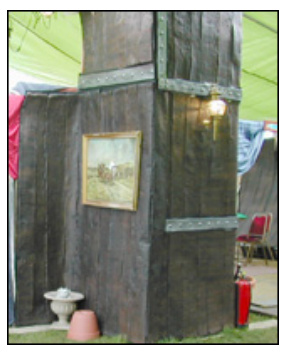

(6)

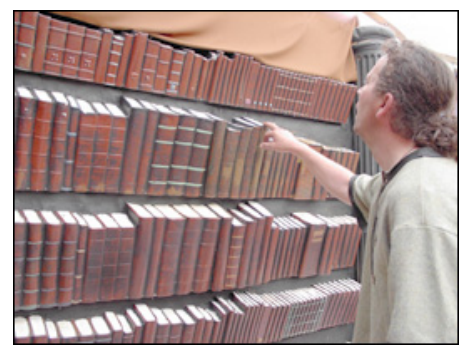

(7)

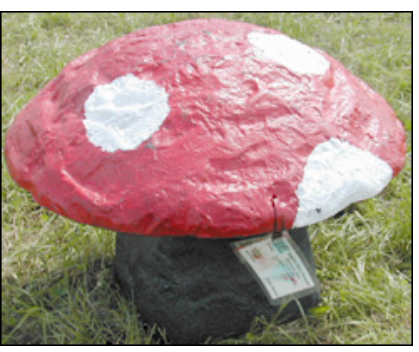

(8)

Fig. 6.-8. Props and physical structures support location-specific interaction and functionality

Players frequently use physical artifacts as props and tools in their role-play, primarily to back up their character roles. Commonly referred to as physical representations, or physreps, they represent game objects with tangible presence and functionality in the game. Mechanisms named lammies (laminated pieces of paper) formalize physreps' functionality in the game. Figure 10 shows an example, an amber talisman

1 We are aware that this is unlikely to make much sense. This device is an illogical construct to logically justify the act of traveling distances in a time-frame that defies laws of physics. 


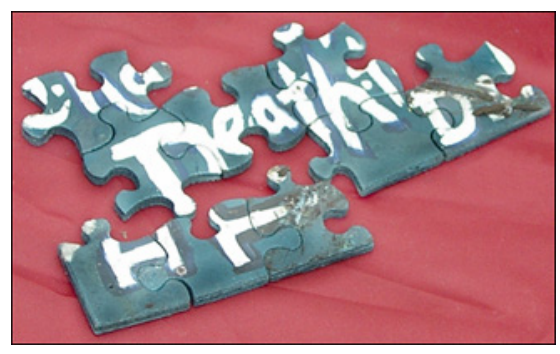

(9)

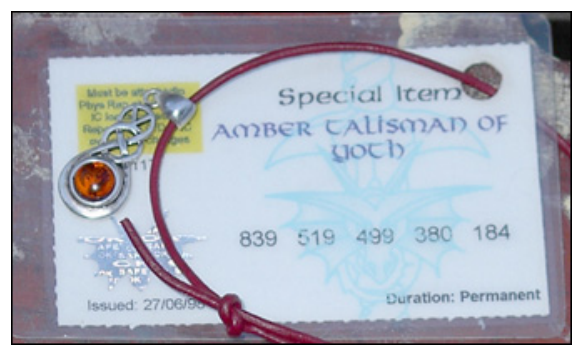

(10)

Fig. 9.-10. Samples of physreps, physical representations of game artifacts

that protects its wearer from certain diseases. Numbers are printed on the 'lammie', making up a codified reference to intrinsic properties such as monetary value, origin, magical nature, and so forth. Players with characters that have the appropriate skills can check these codes against so called 'lore-sheets', a mechanism that manages and sanctifies knowledge in the game. In this fashion, a 'lammie' enables plug-and-play features and offers a way to transform arbitrary objects into official game artifacts.

The most important difference between live role-playing games and computer games is that LRP players interact with and experience the game world directly. The physical environment allows for sophisticated sensory engagement. The players' perception of and navigation through space, and manipulation and organization of artifacts, can be transformed into game-related actions. The physical world interface is not a metaphor for interaction, but the medium for interaction.

\subsection{Monsters as Interface}

The Lorien Trust and many other systems use a supporting cast of characters to interact with the player characters. They may function as opposition and threat for the players to overcome: sometimes through violence, sometimes through politics and diplomacy. The degree of creative and improvisational freedom they have in their roles can vary. These characters have names like monsters, non-player characters $(N P C)$, scripted-or directed player characters, or crew characters. They are part of the game environment, like extras in a movie set, often played by members of the organizing team. The least autonomous are controlled and directed in terms of behavior and motivation - they are told what to do, when and how to do it. They are quick injections into the scenery and the plot - e.g. the beggar that the players pass on their way to the village, or the wise woman that appears at their campfire at night to pass on a message and then disappears to never be encountered again, and so forth.

In contrast, NPCs and other scripted characters may also have a continuous presence amongst the player characters. As such they often have complex roles that are important in the overarching plan for the narrative progression and are often devised by the organizers and assigned to a person to play. While they may have a higher degree of freedom in their roles, they often must report to and accept being controlled by the organizing team. All of these characters add scenery and tangible points of interaction. They are the organizers' most potent, real-time interface to the players, allowing them to seamlessly infuse the game with in-character events on the fly. 


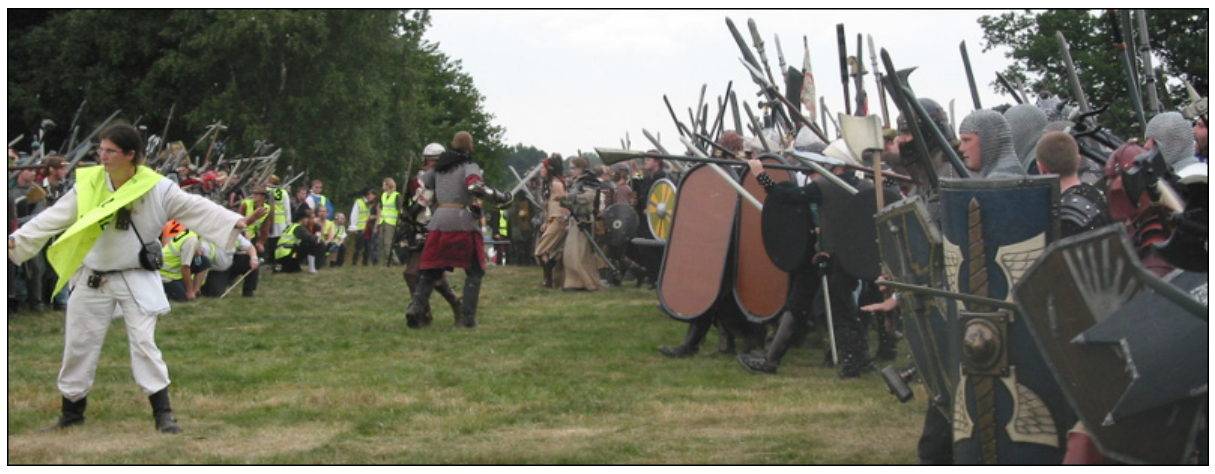

Fig. 11. Battles contributes in part to the experience of personal immediacy

In the Lorien Trust system, most characters carry one weapon or another and many characters have fighter characteristics and skills. It is beyond the scope of this paper to argue for or against the pros and cons of violence or the affect the battle mechanism has on individuals, but it should be noted that the potential threat that armed conflict poses contributes to creating an experience of strong personal immediacy, particularly in the battle situation itself. The presence of this type of threat - the danger of exposing ones character to injury and mortal wounds - is of course a beautifully simple way of maintaining that tension required by the definition of a game. Further, it conditions many of the social interactions, is reflected in the costumes people choose for their characters, and most certainly adds to the spectacle!

\subsection{The 'Magic Circle' in LRP}

The magic circle is a term referring to a place in time and space that game players enter into when they agree to play a game together [13, pp.93-99], marking the beginning and the end of the game. It is also an abstract place that provides context to game actions, and as such allows a safe and lucid place for the players to submit their behavior. This is both a tangible (like the board of a board game) and an intangible (like house rules in a card game) construct in most games. As described by Salen and Zimmerman, special meaning is assigned to objects and to behavior within the magic circle, effectively creating a new reality and a cognitive frame for understanding this new reality. In games that constantly re-negotiate properties of a physical space into properties of a game space, as LRP games do, the magic circle is effectively blurred. To remedy this, LRP games must rely on some core unambiguous principles, or suffer from confused players who fail to distinguish the game from the real world. Costume is of course one of the most powerful mechanisms to signal when a person is playing the game, but lammies, the use of language, hand gestures and such tangible symbols are of equal importance.

Since the magic circle is meant to provide a safe place to submit play behavior, some may argue that the blurred and dissolved magic circle in LRP games poses the danger of anti-social addictions and players mistaking serious real life issues as part of playing a game. It is difficult to fend off those fears when these mistakes occasionally are made. However, in our experience, LRP players are more often than not sensitive to the fact that they are indeed playing a game. Jane McGonigal, describes 
sitive to the fact that they are indeed playing a game. Jane McGonigal, describes this very well when she points out the difference between the 'performance of belief', and the 'suspension of disbelief' [9]. Performing with belief is a social, active, expressive, and externalized act, while the suspension of disbelief is a solitary, passive, submissive, and internalized act. This is something that happens regularly in theatre and in cinemas. Immersive play, she argues, is foremost a desire of gamers for their virtual play to become real and rather than asking "to what extent players come to believe in the fictions they perform, we should ask: To what ends, and through what mechanisms, do players pretend to believe their own performances?"

\section{Implications for Game Design}

LRP games take place in a magical domain somewhere in the cross-sections between imagination, physical reality and fantastic fiction. They offer the kind of immersion that most games and interactive narratives promise as a technical goal but have yet to deliver. In an LRP game there is no physical division between player, character, and narrative, or between the real world and the game world. Some might argue that this level of immersion is the holy grail of interactive entertainment, where the content and the interaction with it is embedded in physical locations and in objects around us, creating a tangible, ubiquitous, and sensual interface to the game activity.

Pervasive games face many design challenges in order to meet the expectations on their ability to immerse players in a game world that is distinct but yet indistinguishable from the real world. It is our position that on the level of interface design, one possible and potentially potent approach begins to reveal itself when observing LRP games. Our study so far has been directed towards understanding the principles by which LRP interfaces contribute to making the game work and we argue that it is because they are believable, tangible, seductive, and part of a ubiquitous game environment. LRP interfaces come together as mechanisms that create social interaction, deliver content, contextualize the game, structure the narrative, and create immersion and engagement. We believe that this points in a direction of great importance to the design of pervasive game interfaces.

\subsection{Ubiquitous: Games in Place}

Mark Weiser's widely accepted definition of ubiquitous computing is the integration of a computational layer with the "fabric of everyday life" [14]. Its most obvious relevance to work on pervasive games is technological - the emphasis on embedding computation in our physical environment. From an interface perspective, ubiquitous computing also provides a compelling model that supports the notion of immersion. The argument that ubiquitous computing environments allow people to concentrate on interacting with each other rather than with computers carries additional appeal that would suggest social and cultural benefits.

Because the immediacy of the physical world is so pertinent, because of LRP games integration with the time and space of the physical environment, and because they incorporate game interfaces that are part of the physical world rather than apart from it, LRP games and the physical environments they are situated in make up some 
of the most powerful examples at hand when considering the design of pervasive games.

\subsection{Tangible: An Interface That Touches}

Tangible user interfaces [5] provide physical form, or embodiment, to digital content and controls, and thereby grant privilege to the sophisticated skills with which we interact with and make sense of the world. The term tangible does not refer only to the physical properties of the interface. It is not sufficient that it is graspable or that it has a presence in the real world. Rather, the word tangible in the context of tangible interfaces emphasizes control and representation of digital information as properties of the same artifact. It is not merely the case that a tangible interface can be touched it also touches you back. In LRP, the physical props, dedicated physical gamelocations, and perhaps most importantly, the characters themselves, are examples of interfaces that grant the players the opportunity to experience game content directly as opposed to abstractly, using the world as a medium for that interaction as opposed to a metaphor.

\subsection{Believable: Lucidity in Representation}

Computer gaming environments are increasingly realistic in their use of the physical world as a model for their game worlds. Many games have as a feature next to photographic graphical representations of the game environment in their attention to detail in scenery. LRP environments are founded on a different attention to detail, where the game world is believable and convincing because there is no separation between the game world and the physical world. We can note that physreps, costume, and props rarely take token shapes or forms, but are instead carefully crafted to convey their dedicated purpose through their physical manifestation. These mechanisms contribute to supporting the performance of belief, helping the player express and display themselves, enabling them to share their performance in a meaningful way with other players. Elaborately dressing the environment with theatrical props and tangible game artifacts, as exemplified by the library setting in figure 6 , is one way to make the players believe in and agree with what happens around them.

\subsection{Magic: Interfaces That Seduce}

While believability is important, what makes LRP worlds spellbinding is that they are typically rendered fantastic rather than realistic. The touch and feel of the game, the magic dust that has been sprinkled over it, the engrossing stories that you partake in when you are inside it, the mind-boggling consequences of your decisions sparking curiosity and seducing you, beckoning you to interact within the game world and with other players. As a simple example, take the puzzle in figure 9, which when solved not only spells out a message, but also functions as a key that unlocks the vessel containing a particularly nasty creature. In this example, the player will know what to do or how to interact with the puzzle, but cannot be certain what the result of that interaction is. The fact that it begins to suggest its functionality - the word "Death" is being 
spelled out when the pieces are put together - is part of encoding this particular artifact's magical message. The puzzle and its initially concealed functionality is part and parcel to the alluring, if not seductive, LRP environment that strengthens players' interest and even commitment to engage with the game world. Interestingly, players are habitually sensitive to the fact that messing with game artifacts often have unexpected effects, which is reflected in a typically curious but careful approach to them.

\section{Conclusion}

There is an unmistakable trend within the human-computer interaction community that points in the direction of pervasive technologies and ubiquitous interface cultures; a trend that is currently making rapid advances on entertainment domain. Future electronic entertainment applications - specifically future computer games and interactive narratives - will move participation and interaction into the physical world. These will be profound gaming experiences in which the real virtuality of the game world is manifest in physical locations and objects around us. This is the holy grail of interactive entertainment - pervasive, tangible, and sensory-intense digital interface design.

Acknowledgements. This research has been supported and financed by Media Lab Europe. A heartfelt Thank You goes out especially to the members of the Story Networks group. Lastly, we want to acknowledge the players in the Lorien Trust LRP system for valuable discussions.

\section{References}

1. Björk, S., Falk, J., Hansson, R., and Ljungstrand, P. Pirates! Using the Physical World as a Game Board. In Proceedings of Interact'01, Tokyo, Japan, 2001.

2. Blast Theory http://www.blasttheory.co.uk/

3. Falk, J. Interfacing the Narrative Experience. In Funology: From Usability to Enjoyment. Blythe, M.A., Overbeeke, K., Monk, A.F., Wright, P.C (eds.), Kluwer Academic Publishers, 2003.

4. Fine, G.A. Shared Fantasy: Role-Playing Games as Social Worlds, The University of Chicago Press, 1983.

5. Ishii, H., and Ullmer, B. Tangible Bits: Towards Seamless Interfaces between People, Bits, and Atoms. In Proceedings of CHI 1997, Atlanta, GA, ACM, 1997.

6. Johnstone, K. Impro: Improvisation and the Theatre. Methuen Publishing Limited, London, UK, 1989.

7. Lorien Trust. http://www.lorientrust.com/

8. Mackay, D. The Fantasy Role-Playing Game - A New Performing Art, McFarland \& Company Inc., 2001

9. McGonigal, J. A Real Little Game: The Performance of Belief in Pervasive Play. In Proceedings of DiGRA's Level-Up, 2003.

10. Murray, J. Hamlet on the Holodeck, pp. 42, MIT Press, 2001.

11. Pan, P., Kastner, C., Crow, D., and Davenport, D. M-Studio: An Authoring Application for Context-Aware Multimedia. In Proceedings of ACM Multimedia 2002, Juan-les-Pins, France, 2002. 
12. Pohjola, M. The Manifesto of the Turku School. In As LARP Grows Up - Theory and Methods in LARP, pp. 32-41, Projektgruppen KP03, 2003. Available at:

http://www.laivforum.dk/kp03_book/classics/turku.pdf

13. Salen, K., Zimmerman, E. Rules of Play. MIT Press, 2004.

14. Weiser, M. The Computer for the $21^{\text {st }}$ Century, Scientific American, pp. 94-110, 1991.

15. Wingård, L., and Fatland, Eirik. Dogma 99 - A programme for the liberation of LARP. Available at: http://fate.laiv.org/dogme99/ 